\title{
Discharge FGF23 level predicts one year outcome in patients admitted with acute heart failure
}

\author{
Giuseppe Vergaro $^{\mathrm{a}, \mathrm{b}, 1}$, Alberto Aimo ${ }^{\mathrm{a}, \mathrm{b}, *, 1}$, Ester Taurino ${ }^{\mathrm{c}}$, Annamaria Del Franco ${ }^{\mathrm{a}, \mathrm{b}}$, Iacopo Fabiani ${ }^{\mathrm{b}}$, \\ Concetta Prontera $^{\mathrm{b}}$, Silvia Masotti ${ }^{\mathrm{b}}$, Veronica Musetti ${ }^{\mathrm{a}, \mathrm{b}}$, Michele Emdin ${ }^{\mathrm{a}, \mathrm{b}}$, Claudio Passino ${ }^{\mathrm{a}, \mathrm{b}}$ \\ a Institute of Life Sciences, Scuola Superiore Sant'Anna, Pisa, Italy \\ b Cardiology Division, Fondazione Toscana Gabriele Monasterio, Pisa, Italy \\ c Emergency Department, Azienda Ospedaliera Universitaria Pisana, Pisa, Italy
}

\section{A R T I C L E I N F O}

\section{Article history:}

Received 18 March 2021

Received in revised form 10 May 2021

Accepted 13 May 2021

Available online 19 May 2021

\section{Keywords:}

FGF23

Acute heart failure

Prognosis

Kidney

\begin{abstract}
A B S T R A C T
Background: Patients with acute heart failure (AHF) show high levels of fibroblast growth factor-23 (FGF23) on admission. We examined if plasma FGF23 changes during an episode of AHF, and if FGF23 holds prognostic significance in this setting.

Methods: Consecutive AHF patients were enrolled. Blood samples were collected on admission and at discharge. Patients were then followed for all-cause death or HF hospitalization.

Results: Patients ( $\mathrm{n}=125$; median age 76 years [interquartile interval $71-83$ ], $63 \% \mathrm{men}$, left ventricular ejection fraction 35\% [25\%-56\%]) had median admission FGF23 $70 \mathrm{ng} / \mathrm{L}$ (47-100), N-terminal pro-B-type natriuretic peptide (NT-proBNP) $5844 \mathrm{ng} / \mathrm{L}$ (2,503-10,468), high-sensitivity troponin T (hs-TnT) $40 \mathrm{ng} / \mathrm{L}$ (25-72), and soluble suppression of tumorigenesis-2 (sST2) $26 \mathrm{ng} / \mathrm{mL}$ (17-37). While other biomarkers decreased, FGF23 increased by $15 \%$ from admission to discharge $(p=0.033)$, with a significant correlation with percent changes in estimated glomerular filtration rate ( rho $=0.306, p=0.001$ ). Over a 12 -month follow-up, 64 patients $(51 \%$ ) experienced the endpoint. They were more often men, older, with higher systolic pulmonary artery pressure (SPAP), higher NT-proBNP, hs-TnT and discharge FGF23. The best FGF23 cut-off at discharge from receiver operating characteristics analysis was $78 \mathrm{ng} / \mathrm{L}$. Both discharge FGF23 and the $78 \mathrm{ng} / \mathrm{L}$ cut-off independently predicted outcome in models including gender, sPAP, age, and 1) admission NT-proBNP, 2) discharge NT-proBNP, 3) admission NTproBNP and hs-TnT, 4) discharge NT-proBNP and hs-TnT. The $78 \mathrm{ng} / \mathrm{L}$ cut-off also refined risk reclassification. Conclusions: During an AHF episode, FGF23 increases from admission to discharge, and patients with higher discharge FGF23 have a higher risk of worse outcome.
\end{abstract}

(c) 2021 Elsevier B.V. All rights reserved.

\section{Introduction}

Since its identification as a phosphaturic agent in the early 2000s (1), fibroblast growth factor 23 (FGF23) has been involved in the control of body metabolism by regulating serum phosphate, parathyroid hormone (PTH), and active vitamin D (2). FGF23 is produced mainly in osteocytes and osteoblasts, although FGF23 expression has been reported in other tissues including the myocardium (3) and kidneys in models of chronic kidney disease (CKD) (4). FGF23 reduces phosphatemia through the inhibition of phosphate reabsorption in the proximal tubule, and decreases plasma PTH and active vitamin D levels (2). The circulating full-length, biologically active hormone, intact FGF23 can be cleaved

\footnotetext{
* Corresponding author at: Institute of Life Sciences, Scuola Superiore Sant'Anna, and Cardiology Division, Fondazione Toscana Gabriele Monasterio, Piazza Martiri della Libertà 33, Pisa 56124, Italy.

E-mail addresses: a.aimo@santannapisa.it, aimoalb@ftgm.it (A. Aimo).

${ }^{1}$ These Authors equally contributed.
}

into inactive $\mathrm{N}$ - and $\mathrm{C}$-terminal fragments by furin, and is at least partially cleared by the kidneys (4). FGF23 has been extensively characterized as a sensitive biomarker of worsening renal function as it rises earlier than most other serum parameters, including creatinine (5). FGF23 is also known to predict poor outcomes in CKD, as higher FGF23 predicts a faster progression to end-stage renal disease and higher cardiovascular morbidity and mortality (6).

High FGF23 is an independent determinant of cardiovascular disease in both CKD patients and in the general population. Elevated FGF23 levels were associated with higher aortic and coronary calcification scores and with arterial stiffness in CKD patients $(5,7)$, and there is evidence of a causal relationship between raised FGF23 and endothelial dysfunction (8). Furthermore, several epidemiologic studies have shown a close relationship between FGF23 and the prevalence of myocardial infarction, left ventricular (LV) hypertrophy and chronic heart failure (HF), in both the CKD and the community population $(9,10)$, although strong experimental evidence of a causal link has not been provided (2). In patients with chronic HF, plasma FGF23 was found to 
independently predict cardiovascular death in a model including age, LV ejection fraction (LVEF), B-type natriuretic peptide (BNP), estimated glomerular filtration rate (eGFR), PTH, calcium, and phosphorus (11). In the same setting, FGF23 predicted all-cause mortality in a model adjusted for age, gender, creatinine clearance, LVEF, New York Heart Association class and N-terminal pro-BNP (NT-proBNP), while not yielding an independent prognostic value for other endpoints including HF hospitalization or the combination of cardiovascular death or HF hospitalization (12).

Patients with acute HF (AHF) have markedly higher plasma FGF23 than healthy controls, including those with preserved renal function, with no correlations observed between FGF23 and eGFR, NT-proBNP, LVEF, or age (3). The level of FGF23 expression in the heart of AHF patients and healthy controls was similar, suggesting that the myocardium does not contribute to the elevation of circulating FGF23 in AHF (3). It is unknown whether plasma FGF23 is affected by the changes in renal function related to AHF and its treatment, and whether FGF23 holds prognostic significance in this setting. We explored these points in a cohort of patients with AHF undergoing an extensive laboratory characterization including previously validated prognostic biomarkers in AHF: NT-proBNP (13), high-sensitivity troponin T (hs-TnT) (13), and soluble suppression of tumorigenesis-2 (sST2) (14).

\section{Methods}

\subsection{Study design}

Consecutive patients admitted for AHF to the Fondazione Toscana Gabriele Monasterio in Pisa, Italy from January 2018 to March 2019 were enrolled. AHF was defined by typical signs and symptoms (e.g., elevated jugular venous pressure, pulmonary crackles and peripheral edema, breathlessness, ankle swelling, fatigue) caused by a structural and/or functional cardiac abnormality, in agreement with European Society of Cardiology (ESC) Guidelines (15). Exclusion criteria were: acute coronary syndrome, recent ( $<3$ months) cardiovascular surgery, acute myocarditis or cardiogenic shock, and inability to provide informed consent.

On admission, each patient underwent a comprehensive cardiovascular workup including clinical history, physical examination, 12-lead electrocardiogram, and transthoracic echocardiogram (TTE). Patients were then managed according to ESC Guidelines (15). For the present analysis, we evaluated only discharged patients (i.e., those surviving the index hospitalization). All patients provided informed consent for this observational study, which was conducted in agreement with the Helsinki Declaration and was approved by the institutional review board (protocol number: 1437; approval date 25/05/2017).

\subsection{Laboratory examination}

Blood samples for FGF23, NT-proBNP, hs-TnT and sST2 were collected on admission and at discharge. Intact FGF23 was assessed with an automated immuno-chemiluminescent sandwich assay (DiaSorin, Saluggia, Italy. NT-proBNP and hs-TnT were assessed by means of electrochemiluminescence monoclonal methods (Roche Diagnostics Italia, Monza, Italy). Plasma sST2 was measured with the Presage ST2 assay on a microtiter plate platform (Critical Diagnostics, San Diego, California, USA). Estimated glomerular filtration rate (eGFR) was calculated through the Chronic Kidney Disease Epidemiology Collaboration (CKDEPI) formula.

\subsection{Echocardiography}

All TTE evaluations were performed by expert sonographers and reviewed by senior cardiologists. The reading was standardized and consistent across years. Standard, two-dimensional TTE images were obtained using a Philips IE33 Ultrasound machine, with X5-1 transducer (Philips Medical Systems, Palo Alto, California, USA). Wall thickness, chamber volumes, and indices of systolic and diastolic function were assessed according to the American Society of Echocardiography and European Association of Cardiovascular Imaging guidelines $(16,17)$.

\subsection{Follow-up}

All patients had visits scheduled at every 3 to 6 months, as clinically indicated. Independent interviewers obtained data from patients, relatives, or general practitioners. The follow-up lasted until October 2020. Information about time and cause of death was retrieved from death certificates, postmortem reports, and general practitioners. The end-point was all-cause death or first HF hospitalization.

\subsection{Statistical analysis}

IBM SPSS Statistics (version 22, 2013) and R statistical software (http://www.r-project.org/, version 3.4.4) were used. Normal distribution was assessed by the Shapiro-Wilk test; variables with normal distribution were presented as means \pm standard deviations, while those with non-normal distribution as medians and interquartile intervals. There were no missing data except for HF therapies at discharge, which were available in 122 patients out of 125 (98\%); missing data were not imputed. Mean differences among groups were evaluated through the unpaired Student $t$-test; changes in biomarkers from admission to discharge were assessed through the paired Student $t$-test. Categorical variables were compared by the Chi-square test with Yates correction. The Spearman's rho coefficient was calculated as a measure of correlation. Cubic spline interpolation was carried out to represent the changes in risk according to biomarker values; 5 knots were considered. The value of discharge FGF23 for which the relative hazard ratio = 1 was chosen as the value corresponding to the inflection point of the curve, above which the slope of the curve becomes steeper. The best cut-off at receiver operating characteristics analysis was searched through the Youden method. At Kaplan-Meier analysis, differences in survival between groups were tested with the log-rank test (MantelCox). NT-proBNP, hs-TnT, sST2 and FGF23 were ln-transformed for inclusion in multivariable Cox regression analysis. The number of variables in the model was chosen based on the one-in-ten event rules. Multicollinearity was searched through the variance inflation factor, with a conservative threshold of 3 . The continuous net reclassification improvement (NRI), integrated discrimination improvement (IDI) and global Chi-square from Cox regression analysis were calculated to assess the added value of discharge FGF23 to a baseline model. Two-tailed $p$ values $<0.05$ were considered significant.

\section{Results}

\subsection{Patient population and outcome}

One hundred twenty-five consecutive patients were evaluated; their main characteristics are reported in Table 1. Patients were aged 76 years (interquartile interval 71-83), and 63\% were men. eGFR was $55 \mathrm{~mL} / \mathrm{min} / 1.73 \mathrm{~m}^{2}$ (38-75), systolic blood pressure was $120 \mathrm{mmHg}$ (105-130), and LVEF was 35\% (25\%-56\%). Over a median follow-up of 12 months (7-18), 64 patients (51\%) experienced the composite of all-cause death or HF hospitalization, with 50 deaths and 30 first HF hospitalizations. Patients experiencing this endpoint were more often men, had a higher estimated systolic pulmonary artery pressure (sPAP), and tended to be elderly $(p=0.051)$. No other significant differences emerged, apart from a higher mean diuretic dose and a more common use of levosimendan (Table 1). 
Table 1

Population characteristics.

\begin{tabular}{|c|c|c|c|c|}
\hline & \multirow[t]{2}{*}{ Whole population $(\mathrm{n}=125)$} & \multicolumn{2}{|c|}{ Death or HF hospitalization } & \multirow[t]{2}{*}{$p$} \\
\hline & & Yes $(n=64,51 \%)$ & No $(\mathrm{n}=61,49 \%)$ & \\
\hline Age (years) & $76(71-83)$ & $79(72-83)$ & $76(68-82)$ & 0.051 \\
\hline Male gender, n (\%) & $79(63)$ & $48(75)$ & $31(51)$ & 0.005 \\
\hline BMI $\left(\mathrm{kg} / \mathrm{m}^{2}\right)$ & $27(24-30)$ & $27(23-29)$ & $26(24-31)$ & 0.647 \\
\hline Ischemic etiology, n (\%) & $28(22)$ & $11(17)$ & $17(28)$ & 0.152 \\
\hline Hypertension, $\mathrm{n}(\%)$ & $72(58)$ & $36(56)$ & $36(59)$ & 0.754 \\
\hline Hypercholesterolemia, n (\%) & $43(34)$ & $27(42)$ & $16(26)$ & 0.060 \\
\hline Diabetes mellitus, n (\%) & $45(36)$ & $25(39)$ & $20(33)$ & 0.465 \\
\hline COPD, n (\%) & $27(22)$ & $16(25)$ & $11(18)$ & 0.344 \\
\hline eGFR $\left(\mathrm{mL} / \mathrm{min} / 1.73 \mathrm{~m}^{2}\right)$ & $55(38-75)$ & $50(35-69)$ & $62(39-89)$ & 0.077 \\
\hline Hemoglobin $(\mathrm{g} / \mathrm{dL})$ & $12(11-13)$ & $12(11-14)$ & $12(11-13)$ & 0.132 \\
\hline LBBB, n (\%) & $53(42)$ & $27(42)$ & $26(43)$ & 0.961 \\
\hline Admission SBP (mmHg) & $120(105-130)$ & $120(100-134)$ & $120(110-130)$ & 0.770 \\
\hline NYHA III-IV, n (\%) & $63(50)$ & $37(58)$ & $26(43)$ & 0.090 \\
\hline \multicolumn{5}{|l|}{ Admission TTE } \\
\hline $\operatorname{LVEF}(\%)$ & $35(25-56)$ & $35(25-55)$ & $38(25-58)$ & 0.947 \\
\hline HFrEF, HFmrEF, HFpEF, n (\%) & $68,18,39(54,14,31)$ & $36,9,19(56,14,30)$ & $32,9,20(53,15,33)$ & 0.910 \\
\hline Diastolic dysfunction grade I, II, III, n (\%) & $78,31,12(62,25,10)$ & $43,12,7(67,19,11)$ & $35,19,5(57,31,8)$ & 0.264 \\
\hline TAPSE $(\mathrm{mm})$ & $16(13-20)$ & $15(13-18)$ & $17(14-21)$ & 0.139 \\
\hline $\mathrm{sPAP}(\mathrm{mmHg})$ & $46(40-53)$ & $50(43-56)$ & $44(39-50)$ & 0.014 \\
\hline \multicolumn{5}{|l|}{ Therapy on admission } \\
\hline Beta-blockers, n (\%) & $90(72)$ & $49(77)$ & $41(67)$ & 0.245 \\
\hline ACEi/ARB, n (\%) & $65(52)$ & $30(47)$ & $35(58)$ & 0.328 \\
\hline Sacubitril/valsartan, n (\%) & $5(4)$ & $2(3)$ & $3(5)$ & 0.609 \\
\hline MRA, n (\%) & $56(45)$ & $33(52)$ & $23(38)$ & 0.119 \\
\hline Furosemide, n (\%) & $112(90)$ & $58(91)$ & $54(89)$ & 0.701 \\
\hline Digoxin, n (\%) & $18(14)$ & $10(16)$ & $8(13)$ & 0.689 \\
\hline ICD, n (\%) & $9(7)$ & $7(11)$ & $2(3)$ & 0.098 \\
\hline CRT, n (\%) & $12(10)$ & $6(9)$ & $6(10)$ & 0.930 \\
\hline Mean furosemide dose (mg) & $98(65-165)$ & $123(70-201)$ & $86(60-138)$ & 0.041 \\
\hline Inotrope therapy, n (\%) & $7(6)$ & $6(1)$ & $1(2)$ & 0.060 \\
\hline Levosimendan, n (\%) & $28(22)$ & $19(28)$ & $9(15)$ & 0.045 \\
\hline \multicolumn{5}{|l|}{ Therapy at discharge } \\
\hline Beta-blockers, n (\%) & $114 / 122(93)$ & $58 / 61(95)$ & $56(92)$ & 0.464 \\
\hline ACEi/ARB, n (\%) & $66 / 122(54)$ & 29/61 (48) & $37(61)$ & 0.541 \\
\hline Sacubitril/valsartan, n (\%) & $9 / 122(7)$ & $6 / 61(10)$ & $3(5)$ & 0.299 \\
\hline MRA, n (\%) & $96 / 122(79)$ & $49 / 61(80)$ & $47(77)$ & 0.658 \\
\hline Furosemide, n (\%) & $122 / 122(100)$ & $61 / 61(100)$ & $61(100)$ & - \\
\hline Digoxin, n (\%) & $22 / 122(18)$ & $13 / 61(21)$ & $9(15)$ & 0.346 \\
\hline
\end{tabular}

The mean furosemide dose was calculated as the sum of intravenous and oral furosemide doses divided by the number of days of diuretic therapy. ACEi/ARB, angiotensin converting enzyme inhibitor/angiotensin receptor blocker; BMI, body mass index; COPD, chronic obstructive pulmonary disease; CRT, cardiac resynchronization therapy; eGFR, estimated glomerular filtration rate; HFmrEF/HFpEF/HFrEF, heart failure with mid-range/preserved/reduced ejection fraction; ICD, implantable cardioverter defibrillator; LBBB, left bundle branch block; LVEF, left ventricular ejection fraction; MRA, mineralocorticoid receptor antagonists; NYHA, New York Heart Association; SBP, systolic blood pressure; sPAP, systolic pulmonary artery pressure; TAPSE, tricuspid annular plane systolic excursion. Significant $p$ values are highlighted in bold.

\subsection{Biomarker levels}

Median values of biomarkers on admission were: FGF23, $70 \mathrm{ng} / \mathrm{L}$ (47-100); NT-proBNP, 5844 ng/L (2503-10,468); hs-TnT, 40 ng/L (25-72); sST2, $26 \mathrm{ng} / \mathrm{mL}$ (17-37). Apart for FGF23, all biomarker levels decreased from admission to discharge: FGF23, 79 ng/L (59-121); NT-proBNP, 3368 ng/L (1,377-8049); hs-TnT, 35 ng/L (21-60); sST2, $20 \mathrm{ng} / \mathrm{mL}(15-29)$. Conversely, FGF23 increased by $15 \%$ ( $-16 \%$ to $+61 \% ; p=0.033)$, and 82 patients (66\%) showed any increase in FGF23. When comparing baseline characteristics and biomarkers on admission and at discharge between patients who displayed $\mathrm{a} \geq 15 \%$ increase in FGF23 and the other patients, the only significant differences were lower FGF23 at baseline, a greater decrease in NT-proBNP, a significantly greater decline in eGFR $(p<0.001)$, and a more pronounced diuretic response (calculated as weight decrease per each $40 \mathrm{mg}$ of intravenous furosemide; Supplemental Table S1). In the whole cohort, a slight decrease in renal function from admission to discharge was observed (delta eGFR: $-1.2 \%$ [ -14.5 to $+11.2 \%]$ ), with a significant correlation with percent changes in FGF23 (rho $=0.306, p$ $=0.001$; Fig. 1a). Accordingly, eGFR decreases became progressively more prominent across quartiles of changes in FGF23 (Fig. 1b). No significant correlations were found with mean furosemide dose $(p=$ 0.099 ), average daily diuresis ( $p=0.888$ ) or percent changes in weight from admission to discharge $(p=0.944)$. Conversely, a weak inverse correlation was observed between diuretic response and percent changes in FGF23 (rho $=0.219, p=0.019$ ); in other words, patients showing a better response to intravenous furosemide experienced a greater increase in FGF23.

\subsection{Prognostic value of discharge FGF23}

The only significant differences in biomarker levels between patients experiencing vs. those not experiencing the endpoint were higher FGF23 at discharge, and higher NT-proBNP and hs-TnT on admission and at discharge (Table 2).

The risk of all-cause death or HF hospitalization increased steeply with increasing discharge FGF23 (Fig. 2). Discharge FGF23 had an area under the curve of 0.629 , with a best cut-off of $78 \mathrm{ng} / \mathrm{L}$ (sensitivity $54 \%$, specificity $59 \%$ ). This cut-off was remarkably close to the inflection point of the spline curve ( $73 \mathrm{ng} / \mathrm{L}$ ), above which the risk increased more 
A

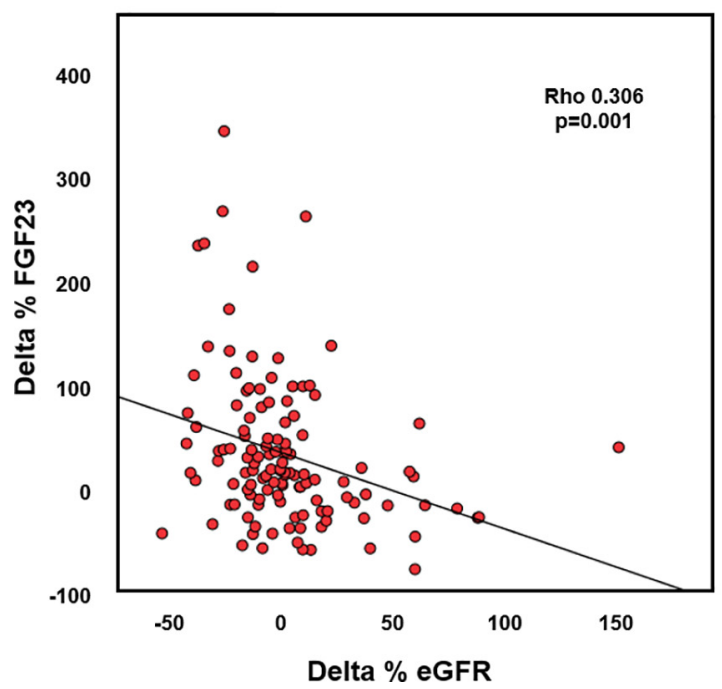

B

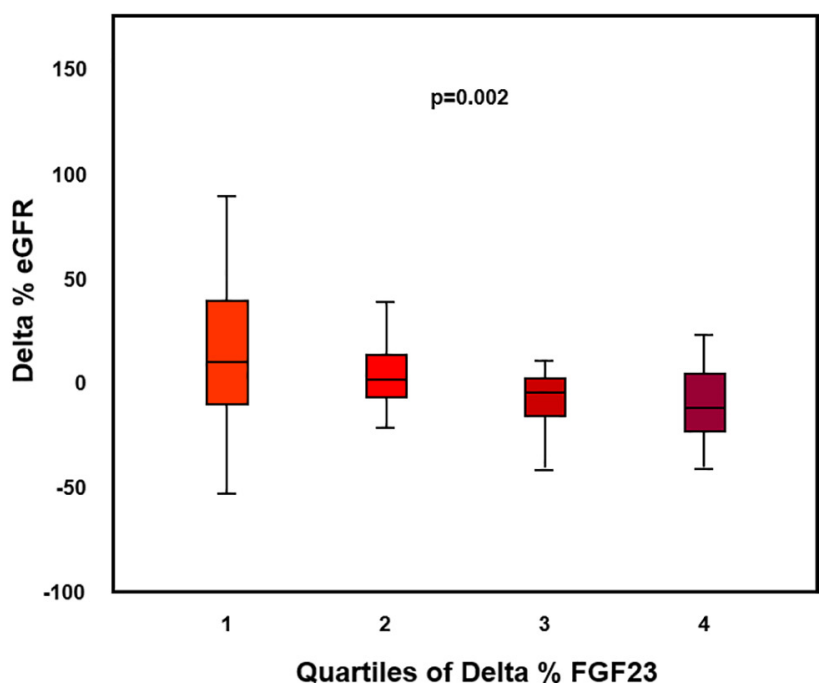

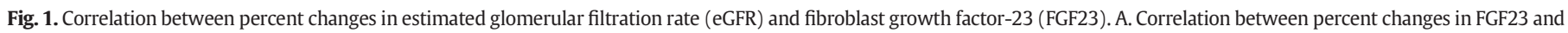
eGFR. B. Values of percent changes in eGFR in each quartile of FGF23 change.

Table 2

Biomarker levels on admission and at discharge according to the occurrence of death or heart failure (HF) hospitalization.

\begin{tabular}{|c|c|c|c|c|}
\hline & \multirow[t]{2}{*}{ Whole population $(\mathrm{n}=125)$} & \multicolumn{2}{|c|}{ Death or HF hospitalization } & \multirow[t]{2}{*}{$p$} \\
\hline & & Yes $(n=64,51 \%)$ & No $(n=61,49 \%)$ & \\
\hline \multicolumn{5}{|l|}{ Admission } \\
\hline FGF23 (ng/L) & $70(47-100)$ & $74(52-117)$ & $69(41-94)$ & 0.144 \\
\hline NT-proBNP (ng/L) & $5844(2503-10,468)$ & $7470(2953-11,828)$ & $4338(2029-8661)$ & 0.038 \\
\hline hs-TnT (ng/L) & $40(25-72)$ & $53(30-88)$ & $34(21-54)$ & 0.002 \\
\hline $\mathrm{sST} 2(\mathrm{ng} / \mathrm{mL})$ & $26(17-37)$ & $27(17-36)$ & $25(19-37)$ & 0.949 \\
\hline \multicolumn{5}{|l|}{ Discharge } \\
\hline FGF23 (ng/L) & $79(59-121)$ & $85(66-129)$ & $71(44-114)$ & 0.013 \\
\hline NT-proBNP (ng/L) & 3368 (1377-8049) & 4457 (1856-9672) & $2689(1130-5247)$ & 0.013 \\
\hline hs-TnT (ng/L) & $35(21-60)$ & $40(27-80)$ & $29(18-47)$ & 0.002 \\
\hline $\mathrm{sST} 2(\mathrm{ng} / \mathrm{mL})$ & $20(15-29)$ & $19(15-34)$ & $21(14-29)$ & 0.607 \\
\hline \multicolumn{5}{|c|}{ Delta \% admission-discharge } \\
\hline FGF23 (\%) & $+15(-16 \text { to }+61)^{a}$ & $+15(-10$ to +81$)$ & $+17(-16$ to +59$)$ & 0.398 \\
\hline NT-proBNP (\%) & $-34(-58 \text { to } 0)^{\mathrm{b}}$ & $-26(-53$ to +4$)$ & $-39(-61$ to -8$)$ & 0.140 \\
\hline hs-TnT (\%) & $-10(-32 \text { to }+9)^{c}$ & $-5(-34$ to +13$)$ & $-13(-30$ to +9$)$ & 0.447 \\
\hline sST2 (\%) & $-20(-44 \text { to }+10)^{d}$ & $-17(-39$ to +10$)$ & $-23(-49$ to +12$)$ & 0.321 \\
\hline
\end{tabular}

FGF23, fibroblast growth factor-23; hs-TnT, high-sensitivity troponin T; NT-proBNP, N-terminal pro-B-type natriuretic peptide; sST2, soluble suppression of tumorigenesis-2. Significant $p$ values are highlighted in bold.

${ }^{\mathrm{a}} p=0.033$.

b $p=0.001$.

$p=0.041$.

d $p=0.065$.

rapidly (Fig. 2). Patients with discharge FGF23 $\geq 78 \mathrm{ng} / \mathrm{L}$ (53\% of the whole study population) had a significantly shorter survival free from the endpoint (Fig. 3), and a 2.6-fold higher risk of the endpoint (odds ratio 2.57 [95\% CI 1.25-5.28]).

We created a prognostic model including male gender and sPAP (i.e., those clinical variables at admission showing significant differences between patients meeting the primary endpoint or not), as well as age (which approached statistical significance; Table 1). Afterwards, we sequentially added admission NT-proBNP and hs-TnT, then discharge NT-proBNP and hs-TnT. Discharge FGF23 displayed an independent prognostic value over all these combinations (Table 3). It also increased Chi-square values, but did not improve 2 metrics of risk reclassification (IDI and NRI; Supplemental Table S1).

The 78 ng/L FGF23 cut-off yielded independent prognostic significance over all the combinations above (Table 4). It also reclassified patient risk, as demonstrated by increases in both NRI and Chi-square values (Supplemental Table S2). Finally, discharge FGF23 and FGF23 $\geq$ $78 \mathrm{ng} / \mathrm{L}$ retained an independent prognostic value over models including admission or discharge sST2 or eGFR (Supplemental Tables S3 and S4).

\section{Discussion}

We report for the first time that plasma FGF23 increases from admission to discharge during an episode of AHF, in parallel with a slight decrease in renal function. Discharge FGF23 independently predicts allcause death or HF hospitalization in prognostic models including admission or discharge NT-proBNP and hs-TnT. We also introduce the $78 \mathrm{ng} / \mathrm{L}$ cut-off of discharge FGF23 as a novel tool for risk stratification, given its strong and independent prognostic value (Central illustration). 


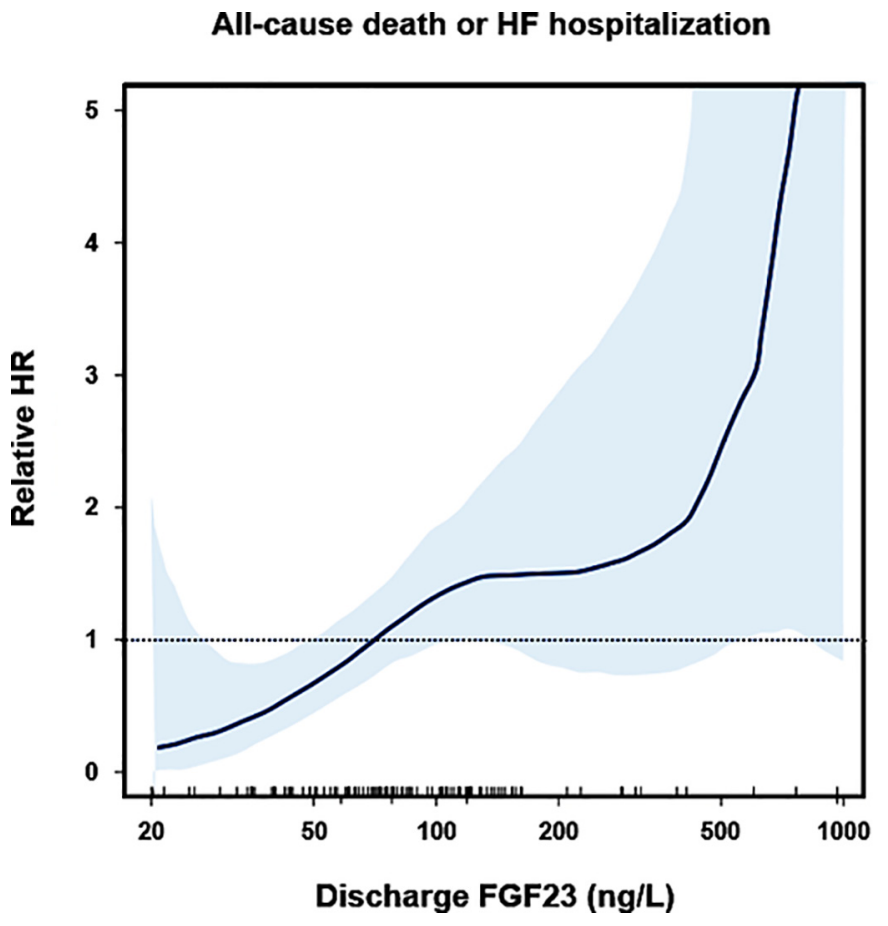

Fig. 2. Discharge fibroblast growth factor-23 (FGF23) and risk of all-cause death or heart failure (HF) hospitalization. Spline curve analysis showing increased hazard ratio (HR) for all-cause death or HF hospitalization for increasing FGF23 levels.
Several biomarkers reflecting different aspects of AHF pathophysiology may predict future disease trajectories. Natriuretic peptides are the most extensively validated tools for risk stratification. A 30\% decrease in NT-proBNP values from admission to discharge is associated with improved hemodynamics and predicts better outcome $(18,19)$. Similarly, higher admission hs-TnT is associated with a greater risk of in-hospital mortality (20), and an increase in hs-TnT during hospitalization predicts adverse short- and long-term outcomes (21). Contrary to other biomarkers, sST2 is not influenced by age, body mass index, renal function or HF etiology (22), and SST2 trends over time provide additive prognostic information over NT-proBNP $(14,23)$.

A surprisingly small number of studies have performed a comprehensive assessment of several HF biomarkers on admission and at discharge, and their relationship with outcome. In this study we carried out this multi-biomarker evaluation, including also FGF23. This last biomarker has been only recently recognized as a pathophysiological player and a prognostic marker in chronic HF with preserved or reduced ejection fraction $(11,12)$. There is evidence that the marked elevation of circulating FGF23 in patients with AHF compared to controls is due to extracardiac production, as both myocardial FGF23 gene expression and immunohistochemistry were similar in either group (3). The significant correlation between percent changes in FGF23 and eGFR points to a relationship between circulating levels and kidney function, possibly due to decreased renal clearance and increased expression in the kidneys (24). In turn, worsening renal function in AHF can be explained by intravenous diuretic therapy (25), and the initiation or up-titration of drugs acting on the renin-angiotensin-aldosterone system. The increasing FGF23 levels in AHF might also be explained by enhanced

\section{All-cause death or HF hospitalization}

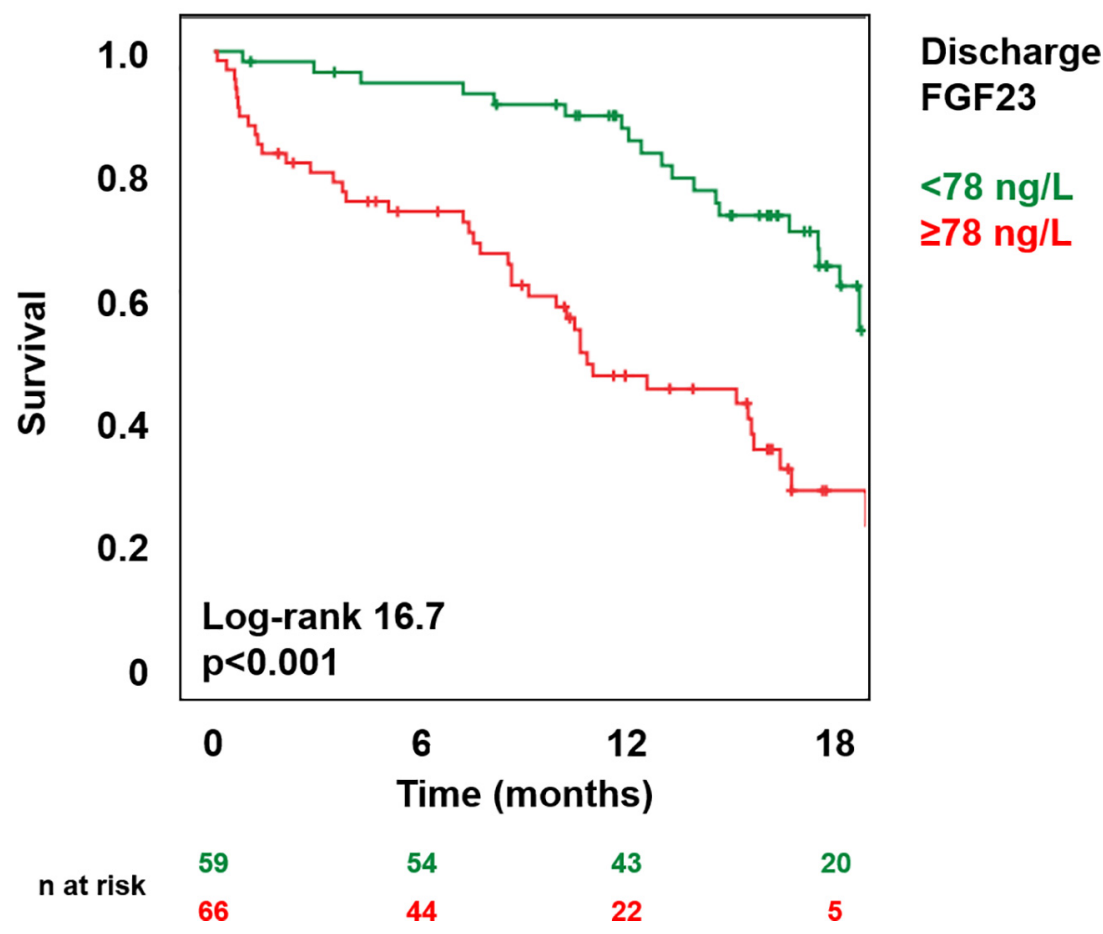

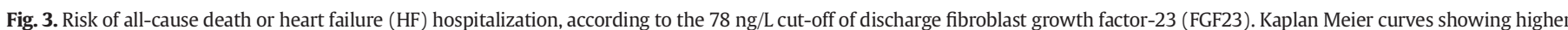
rates of all-cause death or HF hospitalization in patients with FGF23 $\geq 78 \mathrm{ng} / \mathrm{L}$ compared to those with FGF23 $<78 \mathrm{ng} / \mathrm{L}$. 
Table 3

Independent prognostic value of discharge fibroblast growth factor-23 (FGF23): multivariable Cox regression analysis.

\begin{tabular}{|c|c|c|c|c|c|c|c|c|}
\hline & $p$ & $\mathrm{HR}(95 \% \mathrm{CI})$ & $p$ & $\mathrm{HR}(95 \% \mathrm{CI})$ & $p$ & $\mathrm{HR}(95 \% \mathrm{CI})$ & $p$ & $\mathrm{HR}(95 \% \mathrm{CI})$ \\
\hline Male gender & 0.009 & $2.26(1.23-4.15)$ & 0.026 & $2.02(1.09-3.77)$ & 0.012 & $2.13(1.18-3.86)$ & 0.028 & $1.97(1.08-3.60)$ \\
\hline Admission sPAP & 0.312 & - & 0.174 & - & 0.305 & - & 0.180 & - \\
\hline Age & 0.394 & - & 0.729 & - & 0.434 & - & 0.713 & - \\
\hline Admission NT-proBNP & 0.159 & - & 0.820 & - & - & & - & \\
\hline Admission hs-TnT & - & & 0.066 & - & & & & \\
\hline Discharge NT-proBNP & & & - & & 0.055 & - & 0.378 & - \\
\hline Discharge hs-TnT & & & & & - & & 0.091 & - \\
\hline Discharge FGF23 & 0.008 & $1.45(1.10-1.90)$ & 0.013 & $1.40(1.07-1.83)$ & 0.008 & $1.43(1.10-1.86)$ & 0.028 & $1.36(1.03-1.78)$ \\
\hline
\end{tabular}

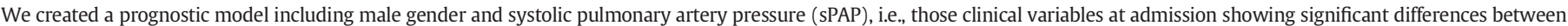

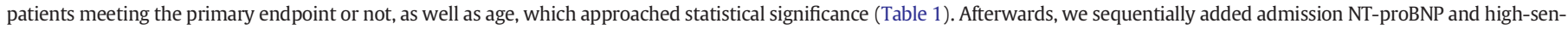

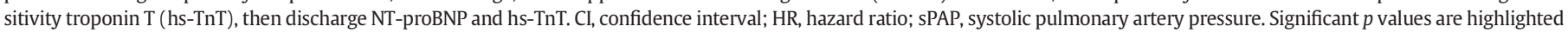
in bold.

Table 4

Independent prognostic value of the discharge fibroblast growth factor-23 (FGF23) $78 \mathrm{ng} / \mathrm{L}$ cut-off.

\begin{tabular}{|c|c|c|c|c|c|c|c|c|}
\hline & $p$ & $\operatorname{HR}(95 \% \mathrm{CI})$ & $p$ & $\mathrm{HR}(95 \% \mathrm{CI})$ & $p$ & $\operatorname{HR}(95 \% \mathrm{CI})$ & $p$ & $\mathrm{HR}(95 \% \mathrm{CI})$ \\
\hline Male gender & 0.007 & $2.30(1.26-4.20)$ & 0.020 & $2.08(1.12-3.85)$ & 0.008 & $2.22(1.23-4.00)$ & 0.020 & $2.05(1.12-3.75)$ \\
\hline Admission sPAP & 0.282 & - & 0.141 & - & 0.278 & - & 0.170 & - \\
\hline Age & 0.432 & - & 0.905 & - & 0.537 & - & 0.800 & - \\
\hline Admission NT-proBNP & 0.183 & - & 0.960 & - & & & & \\
\hline Admission hs-TnT & - & & 0.035 & $1.57(1.03-2.38)$ & & & & \\
\hline Discharge NT-proBNP & & & - & & 0.031 & $1.29(1.02-1.63)$ & 0.270 & - \\
\hline Discharge hs-TnT & & & & & - & & 0.157 & - \\
\hline Discharge FGF23 $\geq 78 \mathrm{ng} / \mathrm{L}$ & 0.003 & $2.36(1.34-4.15)$ & 0.002 & $2.41(1.37-4.26)$ & 0.002 & $2.42(1.40-4.18)$ & 0.005 & $2.23(1.27-3.92)$ \\
\hline
\end{tabular}

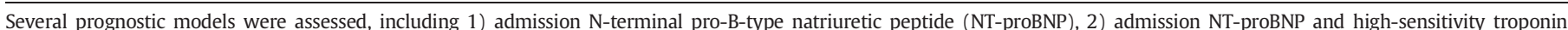

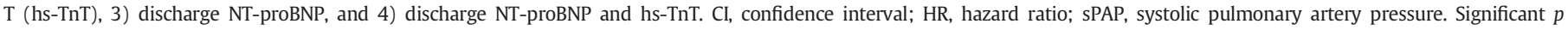
values are highlighted in bold.

bone production stimulated by bone hypoperfusion and hypoxia, as activation of hypoxia-inducible factor $1 \alpha$ (HIF1 $\alpha$ ) has been shown to induce a pronounced increase in FGF23 expression in both primary osteocytes and an osteoblastic cell line (26). In agreement with this hypothesis, patients with acute myocardial infarction complicated by cardiogenic shock show a dramatic increase in FGF23, which is much more pronounced than in uncomplicated acute myocardial infarction (27).

We measured FGF23 through with a fully automated immunoassay approved for clinical use, showing excellent analytical characteristics, and measuring the intact form of FGF23, which has a stronger biological activity than the C-terminal fragment of FGF23 measured in previous studies (28). We report that FGF23 displays a significant 15\% increase from admission to discharge, while NT-proBNP, hs-TnT and SST2 decreased by $34 \%, 10 \%$ and $20 \%$. Interestingly, patients with a significant $30 \%$ decrease in NT-proBNP had also a greater increase in FGF23 than the other patients. Based on these findings, we may hypothesize that a rise in FGF23 denotes a more prominent decongestion in response to AHF treatment. On the other hand, patients reaching particularly high FGF23 values at discharge might also be those with more pronounced bone hypoperfusion and renal dysfunction during the acute phase, and then greater hemodynamic impairment and higher risk of CKD progression, and ultimately a worse prognosis. This hypothesis may explain the relationship between higher discharge FGF23 and worse prognosis, but confirmation is needed. Indeed, a clear relationship between changes in renal function and FGF23 did not clearly emerge from this analysis, possibly because of the small patient number.

Discharge FGF23 emerged as an outcome predictor independent from admission or discharge NT-proBNP, either alone or together with hs-TnT, while sST2 seemed less predictive. We also identified the $78 \mathrm{ng} / \mathrm{L}$ FGF23 cut-off as an independent predictor. The association of FGF23 with specific pathways of organ damage may explain its additive predictive value over other established prognosticators in AHF. The most direct implications of our results are clear, namely that patients with higher FGF23 or FGF23 $\geq 78 \mathrm{ng} / \mathrm{L}$ are at higher risk and might then benefit from a closer follow-up and an enhanced therapeutic effort. Dedicated studies are warranted to test the prognostic benefit of a
FGF23-guided strategy, and possibly also the efficacy of therapies lowering FGF23, such as cinacalcet (a calcimimetic agent that reduces plasma FGF23 and decreases the rates of cardiovascular death and major cardiovascular events in haemodialysis patients) (29) and burosumab, a monoclonal antibody targeting FGF23 recently tested in patients with X-linked hypophosphatemia (30).

Several limitations must be acknowledged to this preliminary, hypothesis-generating study. This was a relatively small, single-center cohort, although all patients had FGF23, NT-proBNP, hs-TnT and sST2 dosed on admission and at discharge, and no patient was lost to follow-up. The limited number of events did not allow to assess other clinically relevant endpoints, such as cardiovascular death, HF hospitalization, or their combination. Finally, we did not assess the performance of FGF23 on top of models with mostly clinical variables, such as the Seattle Heart Failure Model, or the Meta-Analysis Global Group in Chronic Heart Failure (MAGGIC) score, and we did not examine the effects of therapies during the acute phase or after discharge.

In conclusion, absolute FGF23 and FGF23 $\geq 78 \mathrm{ng} / \mathrm{L}$ on discharge both yield independent prognostic value for all-cause death or HF hospitalization in patients with AHF, regardless of admission or discharge NT-proBNP and hs-TnT.

\section{Central illustration}

Fibroblast growth factor-23 (FGF23) and its prognostic value in acute heart failure (AHF).

N-terminal pro-B-type natriuretic peptide (NT-proBNP), highsensitivity troponin $\mathrm{T}$ (hs-TnT), and soluble suppression of tumorigenesis-2 (sST2) which have cardiac or, in the case of sST2, extracardiac production by lung and vessel, decrease during a AHF hospitalization. Conversely, an increase in FGF23 is observed, possibly following increased peripheral synthesis by kidneys and bone.

\section{Funding}

None. 


\section{Contributorship statement}

Giuseppe Vergaro, Alberto Aimo: conceptualization, data curation, formal analysis, writing - original draft; Ester Taurino, Annamaria Del Franco, Iacopo Fabiani: data curation; Concetta Prontera, Silvia Masotti, Veronica Musetti, Michele Emdin, Claudio Passino: data curation, writing - review and editing. Giuseppe Vergaro, Alberto Aimo: responsible for the overall content as guarantors.

\section{Declaration of Competing Interest}

None.

\section{Appendix A. Supplementary data}

Supplementary data to this article can be found online at https://doi. org/10.1016/j.ijcard.2021.05.028.

\section{References}

[1] ADHR Consortium, Autosomal dominant hypophosphataemic rickets is associated with mutations in FGF23, Nat. Genet. 26 (2000) 345-348.

[2] X. Lu, M.C. Hu, Klotho/FGF23 axis in chronic kidney disease and cardiovascular disease, Kidney Dis. 3 (2017) 15-23.

[3] I.A. Andersen, B.K. Huntley, S.S. Sandberg, D.M. Heublein, J.C. Burnett Jr., Elevation of circulating but not myocardial FGF23 in human acute decompensated heart failure, Nephrol. Dial. Transplant. 31 (2016) 767-772.

[4] A.J. van Ballegooijen, E.P. Rhee, S. Elmariah, I.H. de Boer, B. Kestenbaum, Renal clearance of mineral metabolism biomarkers, J. Am. Soc. Nephrol. 27 (2016) 392-397.

[5] L. Desjardins, S. Liabeuf, C. Renard, et al., FGF23 is independently associated with vascular calcification but not bone mineral density in patients at various CKD stages, Osteop. Intern. 23 (2012) 2017-2025.

[6] D. Fliser, B. Kollerits, U. Neyer, et al., Fibroblast growth factor 23 (FGF23) predicts progression of chronic kidney disease: the mild to moderate kidney disease (MMKD) study, J. Am. Soc. Nephrol. 18 (2007) 2600-2608.

[7] M. Zhang, J. Yan, M. Zhu, Z. Ni, Fibroblast growth factor 23 predicts coronary calcification and poor prognosis in patients with chronic kidney disease stages 3-5D, Ann. Clin. Lab. Sci. 45 (2015) 17-22.

[8] N. Silswal, C.D. Touchberry, D.R. Daniel, et al., FGF23 directly impairs endotheliumdependent vasorelaxation by increasing superoxide levels and reducing nitric oxide bioavailability, Am. J. Physiol. Endocrinol. Metab. 307 (2014) E426-E436.

[9] M. Sarmento-Dias, C. Santos-Araújo, R. Poínhos, et al., Fibroblast growth factor 23 is associated with left ventricular hypertrophy, not with uremic vasculopathy in peritoneal dialysis patients, Clin. Nephrol. 85 (2016) 135-141.

[10] M.A. Mirza, A. Larsson, H. Melhus, L. Lind, T.E. Larsson, Serum intact FGF23 associate with left ventricular mass, hypertrophy and geometry in an elderly population, Atherosclerosis. 207 (2009) 546-551.

[11] D. Gruson, T. Lepoutre, J.M. Ketelslegers, J. Cumps, S.A. Ahn, M.F. Rousseau, Cterminal FGF23 is a strong predictor of survival in systolic heart failure, Peptides. 37 (2012) 258-262.
[12] M. Plischke, S. Neuhold, C. Adlbrecht, et al., Inorganic phosphate and FGF-23 predict outcome in stable systolic heart failure, Eur. J. Clin. Investig. 42 (2012) 649-656.

[13] A. Aimo, J.L. Januzzi Jr., C. Mueller, et al., Admission high-sensitivity troponin T and NT-proBNP for outcome prediction in acute heart failure, Int. J. Cardiol. 293 (2019) 137-142.

[14] A. Aimo, G. Vergaro, A. Ripoli, et al., Meta-analysis of soluble suppression of tumorigenicity-2 and prognosis in acute heart failure, JACC Heart Fail. 5 (2017) 287-296.

[15] P. Ponikowski, A.A. Voors, S.D. Anker, et al., 2016 ESC Guidelines for the diagnosis and treatment of acute and chronic heart failure: the task force for the diagnosis and treatment of acute and chronic heart failure of the European Society of Cardiology (ESC) developed with the special contribution of the Heart Failure Association (HFA) of the ESC, Eur. Heart J. 37 (2016) 2129-2200.

[16] R.M. Lang, L.P. Badano, V. Mor-Avi, et al., Recommendations for cardiac chamber quantification by echocardiography in adults: an update from the American Society of Echocardiography and the European Association of Cardiovascular Imaging, J. Am. Soc. Echocardiogr. 28 (2015) 1-39.e14.

[17] P. Lancellotti, C. Tribouilloy, A. Hagendorff, et al., Recommendations for the echocardiographic assessment of native valvular regurgitation: an executive summary from the European Association of Cardiovascular Imaging, Eur. Heart J. Cardiovasc. Imaging 14 (2013) 611-644.

[18] D. Logeart, G. Thabut, P. Jourdain, et al., Predischarge B-type natriuretic peptide assay for identifying patients at high risk of re-admission after decompensated heart failure, J. Am. Coll. Cardiol. 43 (2004) 635-641.

[19] F. Knebel, I. Schimke, K. Pliet, et al., NT-ProBNP in acute heart failure: correlation with invasively measured hemodynamic parameters during recompensation, J. Card. Fail. 11 (2005) S38-S41.

[20] G.M. Felker, V. Hasselblad, W.H. Tang, et al., Troponin I in acute decompensated heart failure: insights from the ASCEND-HF study, Eur. J. Heart Fail. 14 (2012) 1257-1264.

[21] Y. Xue, P. Clopton, W.F. Peacock, A.S. Maisel, Serial changes in high-sensitive troponin I predict outcome in patients with decompensated heart failure, Eur. J. Heart Fail. 13 (2011) 37-42.

[22] A. Aimo, J.L. Januzzi Jr., A. Bayes-Genis, et al., Clinical and prognostic significance of sST2 in heart failure: JACC review topic of the week, J. Am. Coll. Cardiol. 74 (2019) 2193-2203.

[23] L.C. van Vark, I. Lesman-Leegte, S.J. Baart, et al., Prognostic value of serial ST2 measurements in patients with acute heart failure, J. Am. Coll. Cardiol. 70 (2017) 2378-2388.

[24] H. Sugiura, A. Matsushita, M. Futaya, et al., Fibroblast growth factor 23 is upregulated in the kidney in a chronic kidney disease rat model, PLoS One 13 (2018), e0191706,

[25] D.E. Leaf, K.A. Jacob, A. Srivastava, et al., Fibroblast growth factor 23 levels associate with Aki and death in critical illness, J. Am. Soc. Nephrol. 28 (2017) 1877-1885.

[26] J.M. Hum, E.L. Clinkenbeard, C. Ip, et al., The metabolic bone disease associated with the Hyp mutation is independent of osteoblastic HIF1 $\alpha$ expression, Bone Rep. 6 (2017) 38-43.

[27] J. Pöss, F. Mahfoud, S. Seiler, et al., FGF-23 is associated with increased disease severity and early mortality in cardiogenic shock, Eur. Heart J. Acute Cardiovasc. Care 2 (2013) 211-218.

[28] P. Kocełak, M. Olszanecka-Glinianowicz, J. Chudek, Fibroblast growth factor 23structure, function and role in kidney diseases, Adv. Clin. Exp. Med. 21 (2012) 391-401.

[29] S.M. Moe, G.M. Chertow, P.S. Parfrey, et al., Cinacalcet, fibroblast growth factor-23, and cardiovascular disease in hemodialysis: the evaluation of Cinacalcet $\mathrm{HCl}$ therapy to lower cardiovascular events (EVOLVE) trial, Circulation 132 (2015) 27-39.

[30] T.O. Carpenter, M.P. Whyte, E.A. Imel, et al., Burosumab therapy in children with Xlinked hypophosphatemia, New Engl. J. Med. 378 (2018) 1987-1998. 